\title{
Regulation of iron homeostasis by the hypoxia-inducible transcription factors (HIFs)
}

\author{
Carole Peyssonnaux, ${ }^{1,2}$ Annelies S. Zinkernagel, ${ }^{2}$ Reto A. Schuepbach, ${ }^{3}$ Erinn Rankin, ${ }^{4}$ \\ Sophie Vaulont, ${ }^{5,6}$ Volker H. Haase, ${ }^{4}$ Victor Nizet, ${ }^{2}$ and Randall S. Johnson ${ }^{1}$
}

\begin{abstract}
${ }^{1}$ Molecular Biology Section, Division of Biological Sciences, and 2Department of Pediatrics, School of Medicine, UCSD, La Jolla, California, USA. ${ }^{3}$ Department of Immunology, The Scripps Research Institute, La Jolla, California, USA. ${ }^{4}$ Department of Medicine, University of Pennsylvania School of

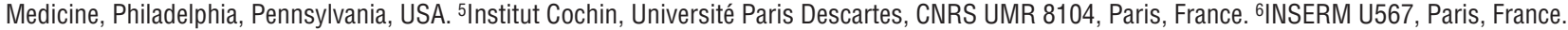

\begin{abstract}
Iron is essential for many biological processes, including oxygen delivery, and its supply is tightly regulated. Hepcidin, a small peptide synthesized in the liver, is a key regulator of iron absorption and homeostasis in mammals. Hepcidin production is increased by iron overload and decreased by anemia and hypoxia; but the molecular mechanisms that govern the hepcidin response to these stimuli are not known. Here we establish that the von Hippel-Lindau/hypoxia-inducible transcription factor (VHL/HIF) pathway is an essential link between iron homeostasis and hepcidin regulation in vivo. Through coordinate downregulation of hepcidin and upregulation of erythropoietin and ferroportin, the VHL-HIF pathway mobilizes iron to support erythrocyte production.
\end{abstract}

\section{Introduction}

Iron metabolism and homeostasis are dependent on the proper regulation of hepcidin, a small antimicrobial peptide expressed in the liver that has only recently been cloned and characterized (1). Genetic studies in mice lacking or overexpressing hepcidin suggest that this peptide inhibits both iron absorption in the small intestine and the release of recycled iron from macrophages $(2,3)$. To limit iron export, hepcidin blocks ferroportin, the sole known iron exporter, by binding to it and causing its internalization and degradation (1). Hepcidin upregulation in response to chronic inflammatory stimuli (e.g., cancer, infection, autoimmunity) leads to the anemia of chronic disease (4-7), while hepcidin downregulation is associated with the iron overload disorder of hemochromatosis (8-11).

Given the links among oxygen transport, erythropoiesis, and iron metabolism, there are potential associations between the physiology of hypoxic response and the control of iron availability. To gain insight into potential hypoxia-dependent molecular mechanisms that might control hepcidin expression in iron homeostasis, we examined transcriptional control of the hypoxic response in the liver by the hypoxia-inducible transcription factors (HIFs), key elements in the response to hypoxia (12). We hypothesized that this pathway could play a role in hepcidin regulation because: (a) iron deprivation and hypoferric anemia lead to poor tissue oxygenation; and (b) HIF levels are partially regulated by the tumor suppressor protein von Hippel-Lindau (VHL). The VHL protein regulates turnover of components of the HIF transcription factors through a process that requires molecular iron.

HIF is a heterodimer whose expression is regulated posttranslationally. Three regulatory HIF subunits have been cloned and named HIF-1 $\alpha$, HIF-2 $\alpha$ (13-15), and HIF-3 $\alpha$ (16). In the presence

Nonstandard abbreviations used: ARNT, aryl hydrocarbon receptor nuclear translocator; ChIP, chromatin IP; DFO, desferrioxamine mesylate; EPO, erythropoietin; HIF, hypoxia-inducible transcription factor; HRE, hypoxia-response element; PHD, prolyl hydroxylase; VHL, von Hippel-Lindau.

Conflict of interest: The authors have declared that no conflict of interest exists. Citation for this article: J. Clin. Invest. 117:1926-1932 (2007). doi:10.1172/JCI31370. of oxygen, the regulatory subunits are modified by iron-dependent prolyl hydroxylases (PHDs) and are then degraded through the ubiquitin/proteasome pathway via its interaction with VHL. Under hypoxia or following iron chelation, PHD activity is inhibited. The regulatory subunit then accumulates and translocates into the nucleus, where it binds to aryl hydrocarbon receptor nuclear translocator/HIF-1 $\beta$ (ARNT/HIF-1 $\beta$ ), which is constitutively expressed. The heterodimeric HIF binds to the hypoxia-response elements (HREs) of target gene regulatory sequences, resulting in the transcription of genes implicated in the control of metabolism and angiogenesis, as well as apoptosis and cellular stress (12). Erythropoietin (EPO) is one of these direct target genes.

In this study, we establish HIF as an essential link between iron fluctuation and hepcidin regulation. The fact that hepcidin, ferroportin, and EPO under the control of VHL/HIF pathway allows a coordinated response for controlling iron metabolism and oxygen transport, regulating the processes that provide iron for physiologic processes such as erythropoiesis. These data provide what we believe to be the first molecular evidence for a coordination between the process of iron uptake and hypoxic response.

\section{Results}

HIF-1 $\alpha$ is stabilized in the liver of iron-starved mice. Mutant mice with iron-deficiency anemia or rats fed an iron-deficient diet exhibit lower hepatic hepcidin expression than control animals $(6,17)$. Studies in patients with iron-deficiency anemia have also demonstrated significantly reduced hepcidin levels relative to those of healthy subjects (18).

We observed that iron starvation of mice of the inbred C57BL/6 strain resulted in a strong decrease in hepatic hepcidin mRNA expression compared with that in control mice fed a normal diet (Figure 1A). Mice in this same study had a clear increase in HIF-1 $\alpha$ levels in the liver in response to the iron deficiency (Figure 1B). This demonstrates that HIF- $1 \alpha$ expression in the liver is influenced by dietary iron depletion.

Decreased regulation of hepcidin by nutritional iron when HIF-1 $\alpha$ is deleted in hepatocytes. HIF- $1 \alpha$ was specifically inactivated in hepatocytes by crossing C57BL/ 6 backcrossed HIF- $1 \alpha^{\text {flox/flox mice }}$ 

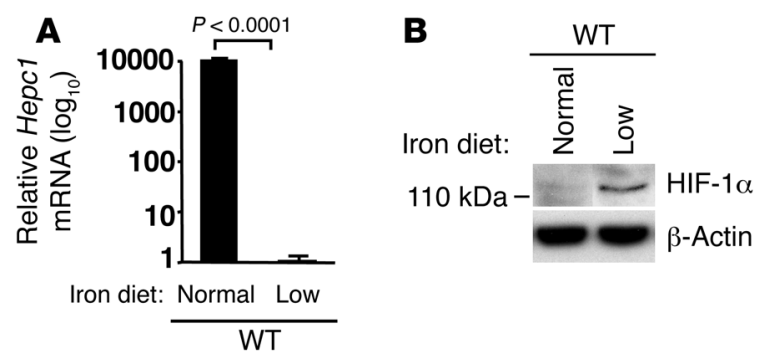

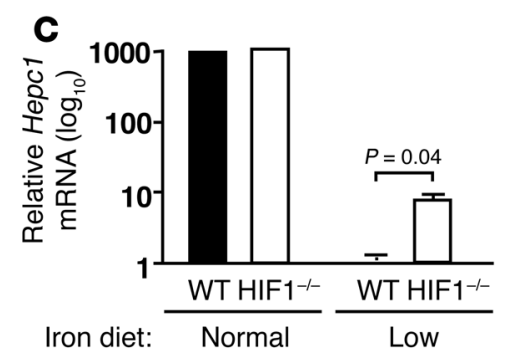

Figure 1

Iron deficiency downregulates hepcidin in an HIF-1-dependent fashion. (A) Hepcidin mRNA level in livers of WT mice under regular or low-iron diet (3 weeks), determined by real-time RT-PCR. Results, normalized to $18 \mathrm{~S}$ ribosomal RNA expression, are expressed as mean $\pm \mathrm{SD}$ ( $n=5$ in each group). (B) HIF-1 expression in liver extracts of iron-starved WT mice by Western blotting. (C) Hepcidin mRNA expression in livers of WT and Albumin-Cre/HIF-1 $\alpha^{\text {floxfllox }}(\mathrm{HIF-1--)})$ iron-starved mice by real-time RT-PCR $(n=8)$. levels of the HIF target gene EPO were increased, reflecting the strong upregulation of EPO mRNA in the liver of AlbuminCre/VHL $L^{\text {flox/flox }}$ compared with WT mice (Figure 2C).

Increased EPO levels are consistent with the increased erythrocytosis and elevated hematocrit observed in the Albumin-Cre/VHL flox/flox mice (Figure 2C). Although total hemoglobin levels were elevated (Figure 2C), mean cell hemoglobin $(\mathrm{MCH})$ was decreased in Albumin-Cre/VHLflox/flox mice compared with WT mice (Figwith Albumin-Cre C57BL/6 transgenic mice. Deletion efficiency in total liver was approximately $70 \%$ as determined by quantitative PCR (data not shown). This indicates a very high rate of hepatocyte deletion, since it is estimated that other cell types, such as endothelial cells and Kupffer cells, make up approximately $30 \%$ of the cells in the liver (19). Under a standard ironrich laboratory diet, HIF-1 $\alpha$ expression was not detectable in the liver (Figure 1B); and no significant differences in hepatic hepcidin expression were observed between WT and Albumin-Cre/ HIF-1 $\alpha^{\text {flox } / \text { flox }}$ mice (Figure 1C).

After 20 days of feeding with an iron-deficient diet, Albumin-Cre/ HIF-1 $\alpha^{\text {flox/flox }}$ mice expressed 10 -fold more hepcidin than WT littermates (Figure 1C), indicating that deletion of HIF-1 $\alpha$ affects the physiological mechanisms governing hepcidin downregulation during nutritional iron deficiency. However, we found that elimination of HIF-1 $\alpha$ alone is insufficient to fully compensate for the hepcidin reduction caused by an iron-deficient diet (Figure 1C), suggesting that other factors also contribute to the observed hepcidin downregulation.

Deletion of VHL in hepatocytes results in polycythemia and iron deficiency. The VHL pathway that controls HIF-1 $\alpha$ levels also regulates HIF-2 $\alpha$, which in turn is thought to have unique roles in regulating liver tumorigenesis and EPO expression (20). The VHL tumor suppressor regulates the oxygen-sensitive turnover of both HIF- $1 \alpha$ and HIF- $2 \alpha$ transcription factors. To investigate the effect of altering the stability of both factors in vivo, we deleted VHL in the liver by crossing C57BL/6 VHL flox/flox mice with Albumin-Cre C57BL/ 6 transgenic mice.

Growth deficiency, polycythemia, and reduced survival occur when VHL is deleted in the liver in a mixed inbred strain genetic background (BALB/c, 129SvJ, and C57BL/6) (21). In the pure C57BL/6 background, mice homozygous for the VHL mutation (Albumin-Cre/VHL flox/flox) in the liver demonstrated alopecia and grew to only half the weight of their WT littermates (Figure 2A), dying between 5 and 7 weeks of age with severe hepatomegaly and splenomegaly (Figure 2B). VHL deletion efficiency in total liver was approximately $50 \%$ as determined by quantitative PCR evaluation of genomic DNA (data not shown).

The livers of Albumin-Cre/VHL flox/flox mice exhibited steatosis (pathologic fat accumulation), with inflammatory cell infiltration as well as foci of necrosis due to fibrin clots (Figure 2B); however, no fibrotic changes were noted. Albumin levels indicative of normal hepatic synthetic function were observed (data not shown). Serum ure 2D). This result indicates a relative iron deficiency in the animals. The observation was corroborated by the hypochromia, poikilocytosis, and microcytosis observed in blood smears from the Albumin-Cre/VHL flox/flox mice (Figure 2D).

Total iron (Figure 2E) and ferritin (Figure 2F) levels in the livers of Albumin-Cre/ $\mathrm{VHL} L^{\text {flox/flox }}$ mice were also greatly reduced relative to those of WT mice. The spleen, another reservoir of iron, was also strongly iron deficient according to Perls Prussian blue staining, as shown in Figure 2G.

The phenotype of the Albumin-Cre/VHL flox/flox mice is similar in some regards to that of humans with Chuvash polycythemia, in which a homozygous mutation $(598 \mathrm{C} \rightarrow \mathrm{T})$ in the VHL gene leads to elevated normoxic levels of HIF-1, serum EPO, and hemoglobin and premature mortality related to cerebral vascular events and peripheral thrombosis $(22,23)$.

HIF-1 binds to the promoter of hepcidin in vivo and reduces its expression in the liver. We detected 2 candidate consensus HREs ([A/G]CGTG) in the murine hepcidin promoter (24) (Figure 3A). We performed a chromatin IP (ChIP) assay on liver tissues extracted from WT and Albumin-Cre/VHL flox/flox mice. Primers flanking HIF- $1 \alpha$ consensus binding sites specifically amplified DNA sequences immunoprecipitated by an HIF-1 $\alpha$ antibody, indicating that HIF- $1 \alpha$ is able to bind the murine hepcidin promoter in vivo (Figure $3 \mathrm{~A}$ ), which supports the assumption of direct regulation of hepcidin by HIF-1.

Examining the human hepcidin promoter sequence, we detected 3 candidate consensus HREs. We performed a ChIP on human HEK293 cells stimulated with the iron chelator desferrioxamine mesylate (DFO) in order to stabilize HIF-1 $\alpha$. In these conditions, primers flanking HIF-1 $\alpha$ consensus binding sites specifically amplified DNA sequence immunoprecipitated by HIF- $1 \alpha$ antibody, showing that HIF-1 $\alpha$ is able to bind the hepcidin promoter in human cells as well as mouse tissues (Figure $3 \mathrm{~B}$ ). We next examined whether the putative classical and conserved HRE (first of 2 HREs arranged as inverted repeats) in the human hepcidin promoter was important for the iron-dependent regulation of hepcidin expression. We cloned the promoter region of the human hepcidin encompassing the HREs into a luciferase reporter construct. The HIF agonist DFO strongly inhibited luciferase activity of this plasmid (pGL3-Hepc/HRE) (Figure 3C), suggesting that HIF binding to the hepcidin promoter suppresses hepcidin gene transcription. To verify that the putative Hepc/HRE located at $-582 \mathrm{bp}$ is the target of repression by HIF, we intro- 
A

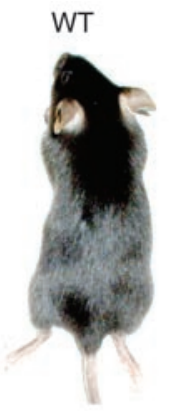

$\mathrm{VHL}^{--}$

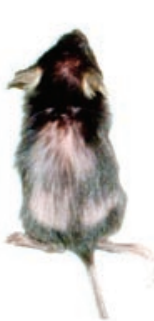

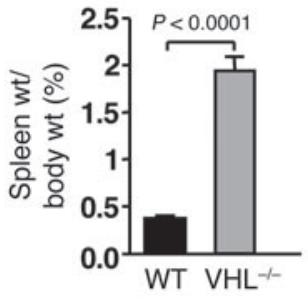

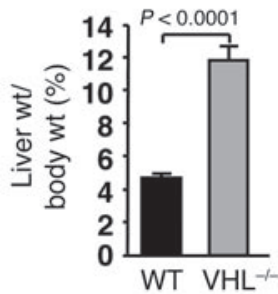

B

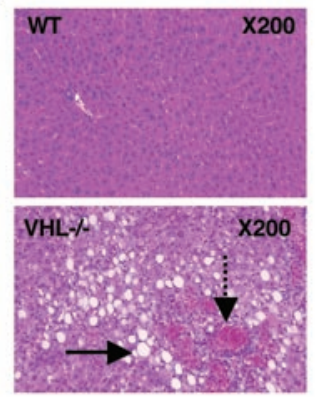

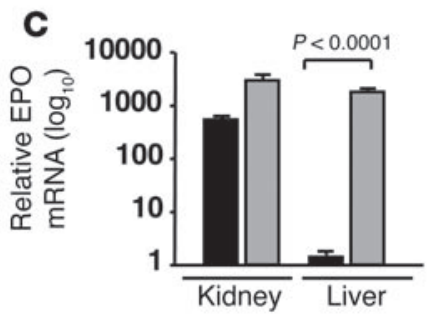

$\operatorname{EPO}(\mathrm{ng} / \mathrm{ml})$
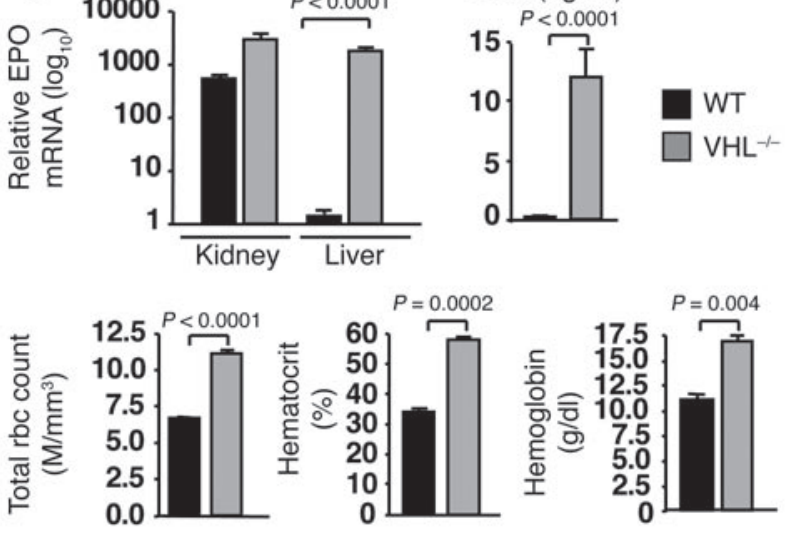

E

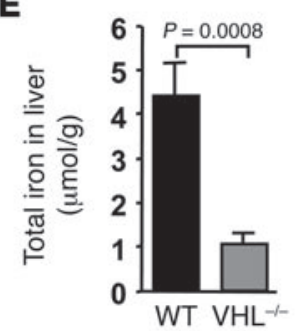

$\mathbf{F}$

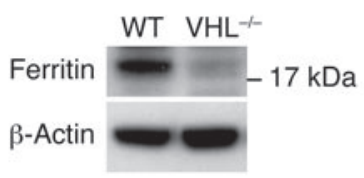

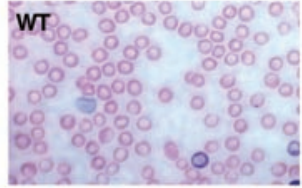
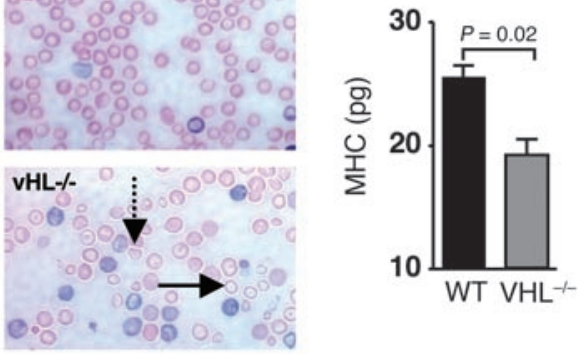

G

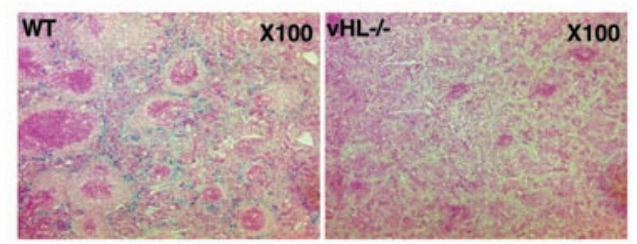

\section{Figure 2}

Albumin-Cre/VHL floxfllox mice develop erythrocytosis and iron deficiency. (A) WT and Albumin-Cre/VHL floxfllox (VHL $\mathrm{VH}^{-/}$) mice (4 weeks old). Right: Spleen and liver weights of 3- to 4-week-old WT and Albumin-Cre/VHL flox/flox mice ( $n=8$ in each group). (B) H\&E stainings of liver sections from WT and Albumin-Cre/VHL floxflox mice. Solid arrow indicates steatosis, dashed arrow inflammatory cell infiltrate. (C) EPO mRNA expression in kidney and liver of WT (black bars) and Albumin-Cre/VHL floxfllox (gray bars) mice by real-time RT-PCR $(n=8)$. EPO, rbc, hematocrit, and hemoglobin levels in blood or serum from 5-week-old mice. $n=8$ in each group. (D) Peripheral blood smears from WT and Albumin-Cre/VHL floxtflox mice. Solid arrow indicates hypochromasia, dashed arrow anisocytosis. Right: mean corpuscular hemoglobin (MCH) of WT and Albumin-Cre/VHL flox/flox mice. Original magnification, $\times 200$. (E) Quantification of liver iron level in WT and Albumin-Cre/VHL flox/flox mice using the method of Torrance et al. (33) ( $n=5$ in each group). (F) Western blot analysis of ferritin in liver extracts from WT and Albumin-Cre/VHLflox/flox mice. (G) Iron staining of splenic sections by Perls Prussian blue. Original magnification, $\times 200$.

duced a point mutation (5'-CAA-TG-3' instead of $5^{\prime}$-CACGTG-3') into this sequence (Figure 3D). The activity of the corresponding plasmid that bears the mutation (pGL3-Hepc/mutHRE) was increased compared with that of the pGL3-Hepc/HRE plasmid. These results demonstrate that the sequence covering the consensus HIF-1 binding site is involved in the downregulation of hepcidin gene transcription by HIF.

We next assessed whether the increased amounts of HIF- $1 \alpha$ in the liver, and its binding to the hepcidin promoter regulate the hepcidin gene in murine tissues. As shown in Figure 3E, Hepc1 mRNA levels measured in the liver of Albumin-Cre/VHLflox/flox mice were markedly decreased compared with those of WT littermates. Analysis of protein levels confirmed that levels of the hepcidin peptide were also lower in Albumin-Cre/VHL flox/flox mice (Figure 3E).

Downregulation of Hepc1 mRNA in Albumin-Cre/VHL flox/flox mice was eliminated, i.e., transcript levels returned to normal values, in Albumin-Cre/VHL flox/flox/ARNTflox/flox double mutant (double-knockout) mice (Figure $3 \mathrm{~F}$ ). These mice have a hepatic deletion of both the VHL gene, whose absence causes increased HIF activity, and the ARNT gene, which is required for dimerization and thus activation of HIF-mediated transcription. The restoration of hepcidin to normal levels in the livers of these double 
A

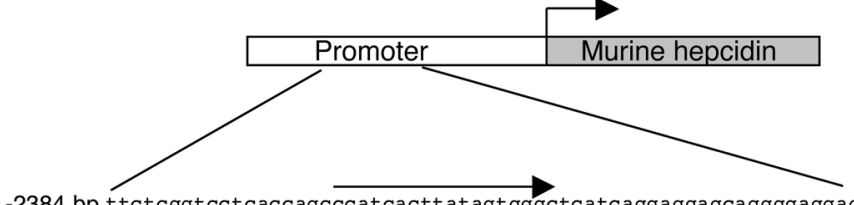

-2384 bp ttctcggtcctcaccagcccatcacttatagtgggctcatcaggaggagcaggggaggag gacagaggccccgctgcccgctgcctccttggtctctagaggtattgagacagggtgctc atgggaaggagggagctttggggatgcgggcettccctggggcttggacctttgcaggga ggccatgcccgccacccctcttgttctggatccctgctcccctctgggtgtgtgggtgtg tgtgtacgtttgggtgtgtgtgtatgdgcgtgtgtgcgtatgtgtgttttaatttctt atgqaaatgqacaaaaatagagagagaggtatttaactgcaataaactgqccagtgtg gccccgccttgtctgtctgtgtgtccatgttgggcatggggttgggtagggttcctaggg -1908 bp tctccetgatatctaagctacagttatactgtagctgtgtgacaatggctgggggcatta

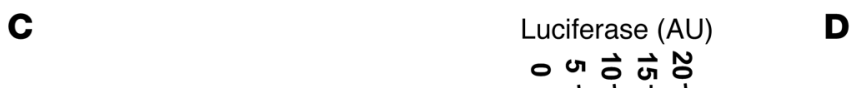

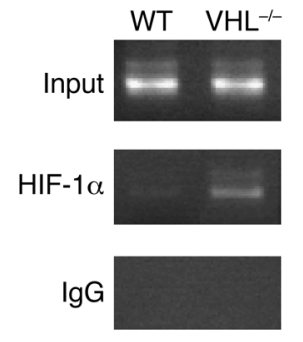

D

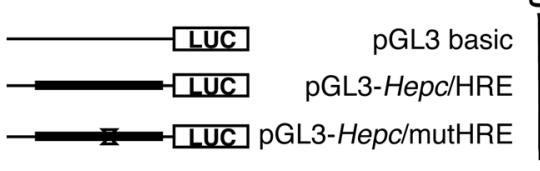

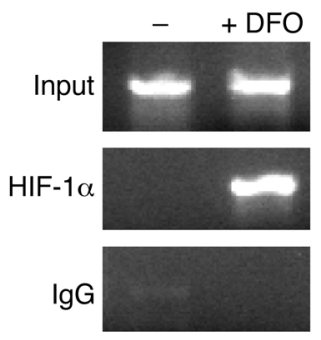

Luciferase (AU)

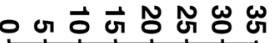

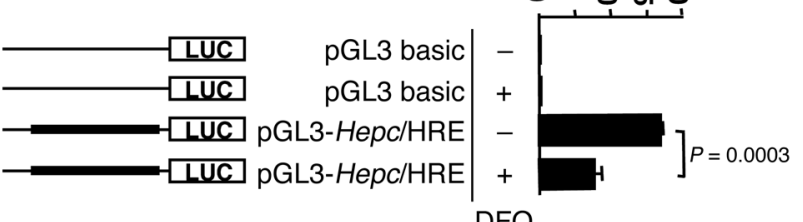

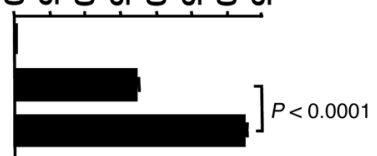

$\mathbf{E}$
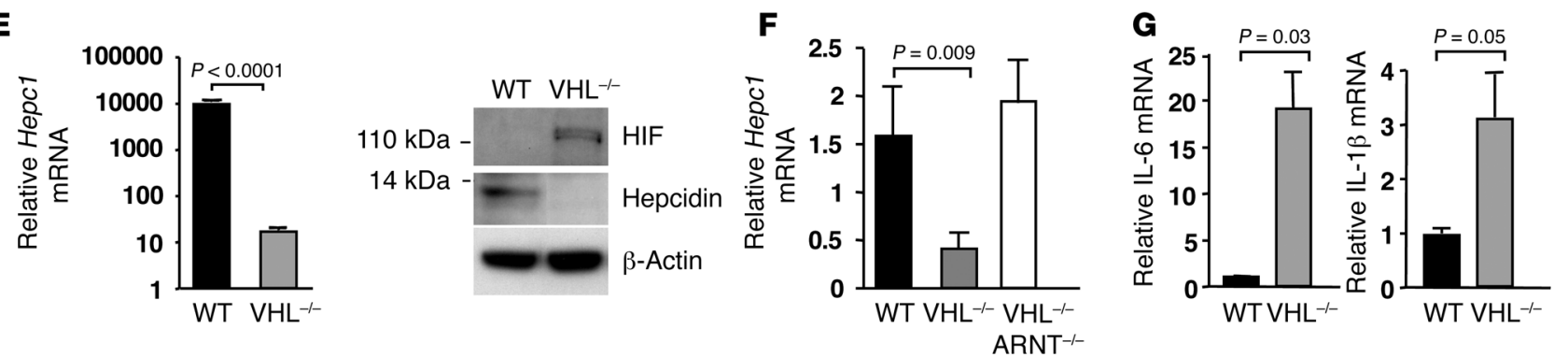

Figure 3

Binding of HIF-1 to the promoter of hepcidin and downregulation of hepcidin in Albumin-Cre/VHL flox/flox. (A) Sequence of murine (C57BL/6) hepcidin promoter; HREs are in bold; arrows indicate primers selected for ChIP. ChIP assay in vivo on liver extracts of WT and Albumin-Cre/ $V H L^{\text {floxfllox }}$ mice. (B) DFO $(150 \mu \mathrm{M})$ induces binding of HIF-1 as shown by ChIP assay. (C) Luciferase-reporter constructs under the control of the regulatory region of the human hepcidin gene. HEK293 cells transiently transfected with pGL3 basic or pGL3-Hepc/HRE vector. (D) The "native" (CCACGTG) and mutated (CAA-TG) HREs (indicated by an X) are shown. HEK293 cells were transiently transfected with pGL3 basic, pGL3-Hepc/HRE, or pGL3-Hepc/mutHRE. (E) Hepcidin mRNA expression in livers of WT and Albumin-Cre/VHLflox/flox by real-time RT-PCR $(n=8)$. HIF-1 and hepcidin expression in liver extracts of WT and Albumin-Cre/VHL flox/flox mice. (F) Hepcidin mRNA expression in livers of WT, Albumin-Cre/VHL floxfllox, and Albumin-Cre/VHL floxflox/ARNT flox/flox $\left(\mathrm{VHL}^{-/-} \mathrm{ANRT}{ }^{-1-}\right)$ mice $(n=4)$. (G) IL-6 and IL-1 $\beta$ mRNA levels in livers of WT and Albumin-Cre/VHL floxflox mice.

mutants demonstrates that hepcidin downregulation in Albumin$\mathrm{Cre} / \mathrm{VH} L^{f l o x} /$ flox mice is specifically due to the stabilization of HIF transcription factors (i.e., HIF- $1 \alpha$ and HIF- $2 \alpha$ ) and not to other functions of the VHL gene.

As Albumin-Cre/VHL flox/flox mice exhibit hepatic inflammation and steatosis (21) (Figure 2C), we examined levels of the proinflammatory cytokine IL-6, which is known to stimulate hepcidin expression and may contribute to the anemia of chronic inflammation (5). Significantly elevated IL-6 and IL-1 levels were found in the Albumin-Cre $/ V H L^{\text {flox/flox }}$ mice (Figure $3 \mathrm{G}$ ), although this change did not prevent the decreased hepcidin expression described above. These findings indicate that IL-6- or IL-1-mediated stimulation of hepcidin expression is subordinate to the suppression caused by increased HIF transcriptional activity.

Upregulation of ferroportin in Albumin-Cre/VHL flox/flox mice. It has recently been shown ex vivo that hepcidin binds ferroportin, the only known iron exporter, thereby trapping iron within enterocytes and macrophages (1). By immunochemis- try, we found significant increases in ferroportin expression in brush border enterocytes of the duodenum of Albumin-Cre/ $V H L^{f l o x} /$ flox mice compared with WT mice (Figure 4A). A likely explanation for this finding is the loss of hepcidin-mediated ferroportin degradation in the Albumin-Cre/VHL flox/flox mice. Analysis of liver sections from Albumin-Cre/VHL flox/flox mice revealed a marked increase in ferroportin in the Kupffer cells of the liver, again, a likely consequence of the decreased hepcidin levels seen in these animals. Interestingly, this was also seen in the Albumin-Cre/VHL flox/flox hepatocytes (Figure 4A).

Western blot analysis confirmed the increase in ferroportin expression in whole liver extracts from Albumin-Cre/VHL flox/flox mice relative to WT mice (Figure 4B). Increased ferroportin levels in the hepatocytes may in part reflect a small but statistically significant increase in hepatic ferroportin mRNA expression detected in Albumin-Cre/VHL flox/flox mice (Figure 4C). While ferroportin expression in the duodenum could result from the decrease in systemic hepcidin expression or from the systemic iron deficiency itself (25), 
A

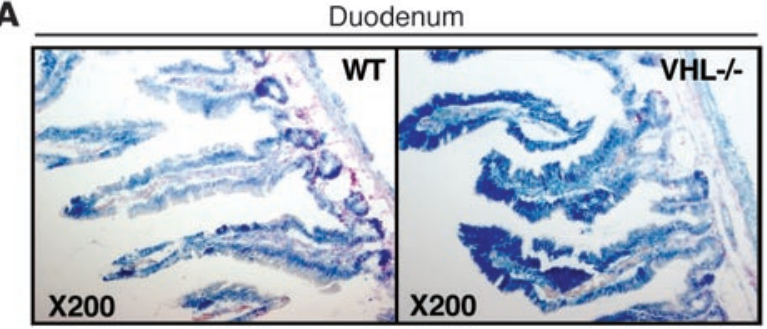

Liver

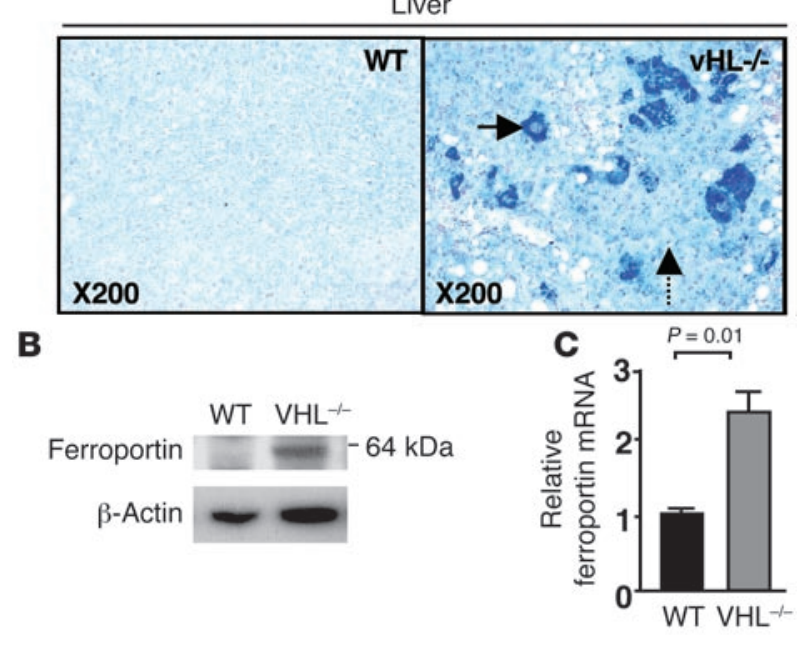

expression of ferroportin in the hepatocyte may reflect a direct tissue-autonomous effect of VHL-deficient cells. Indeed, as previously reported, iron export in hepatocytes is likely independent of the posttranslational regulation of ferroportin by hepcidin $(26,27)$.

\section{Discussion}

Hepcidin is suppressed by both anemia and hypoxia (7). Cellular oxygen sensing and hypoxia-induced transcription are largely mediated by the HIFs. The stabilization of HIF- 1 by iron chelators has been well established in vitro. However, our demonstration of the in vivo induction of HIF-1 by iron deficiency, and the associated downregulation of hepcidin when HIF levels are elevated, suggests that HIF may be one of the missing links between iron homeostasis and hepcidin regulation. The ability of HIF-1 $\alpha$ to bind to and negatively transactivate the hepcidin promoter suggests a direct repressor effect. HIF- $1 \alpha$ has already been reported to repress the transcriptional activity of genes such as alpha-fetoprotein $(A F P)(28)$ and carbamoyl-phosphate synthetase 2, aspartate transcarbamylase, and dihydroorotase (CAD) (29).

Our data show that elimination of HIF-1 alone in adult mice is insufficient to fully compensate for the hepcidin reduction induced by iron depletion. The striking downregulation of hepcidin observed in Albumin-Cre/VHL flox/flox mice, in which both HIF-1 and HIF-2 are stabilized, may suggest a role for HIF-2. HIF-1 may influence embryonic, developmental hepcidin regulation; as Yoon et al. demonstrated, a decrease in TfR in Hif- $1 a^{-/-}$embryos contributes to defects in iron metabolism and consequently an alteration of hepcidin levels (30).

We propose that the VHL/HIF axis serves a central role in coupling iron sensing to iron regulation. In this model, anemia causes decreased tissue oxygenation, which in turn leads to decreased PHD activity and thus decreased VHL-mediated

\section{Figure 4}

Upregulation of ferroportin in Albumin-Cre/VHL flox/flox mice. (A) Immunostaining for ferroportin in duodenum and liver sections from WT and Albumin-Cre/VHL flox/flox mice. Solid arrow indicates a hepatocyte, dashed arrow a Kupffer cell. (B) Ferroportin expression in liver extracts of WT and Albumin-Cre/VHL floxfllox mice. (C) Ferroportin mRNA levels in livers of WT and Albumin-Cre/VHL flox/flox mice. Results are expressed as mean $\pm \mathrm{SD}$ ( $n \geq 4$ in each group); statistical analysis was done using Student's $t$ test (unpaired, 2 tailed).

degradation of HIF- $\alpha$ factors. Increased HIF- $\alpha$ activity causes suppression of hepcidin, increased ferroportin levels, and increased serum iron availability; these in turn allow increased erythropoiesis to be coupled to increased EPO expression.

In support of a role for the HIF axis in regulating this process, we have shown that hepatic deletion of the VHL gene causes decreased hepcidin levels and increased ferroportin expression. Interestingly, in the face of dramatically increased EPO levels and polycythemia, the VHL-deficient animals exhibited microcytosis and low $\mathrm{MCH}$ reminiscent of iron-deficiency. This finding suggests a form of "iron-EPO kinetic imbalance," where the robust proliferation of erythroid precursors creates a demand that outstrips the capacity of the iron delivery system (31). Our model also suggests that inhibition of VHL or PHD activity could represent a novel approach for treatment of anemia of chronic inflammation, since stabilization of HIF prevents hepcidin activation even under the strong stimulus of the proinflammatory cytokine IL- 6 .

It has been proposed that iron balance is conceptually coupled to intestinal iron absorption by 2 mechanisms, the "stores regulator" and the "erythroid regulator," which can be fully characterized only in physiological terms (32). Our results indicate the VHL/HIF axis concomitantly serves both the stores and erythroid regulator pathways, through its responsiveness to oxygen and iron levels and its function as a regulator of hepcidin, ferroportin, and EPO production.

\section{Methods}

Animals. All procedures involving animals were approved by the UCSD Animal Care Committee, which serves to ensure that all federal guidelines concerning animal experimentation are met. Hepatocyte-specific inactivation of HIF- $1 \alpha$ and VHL was achieved by cross-breeding Albumin-Cre transgenic mice (The Jackson Laboratory) with VHL flox/flox mice (backcrossed in a pure $\mathrm{C} 57 \mathrm{BL} / 6$ genetic background). In all experiments, littermates from the same breeding pair were used as controls. Albumin-Cre/VHL flox/flox and Albumin-Cre/VHL flox/flox/ARNT flox/flox mice used in the experiments shown in Figure 3C are in a mixed BALB/c, 129SvJ, and $\mathrm{C} 57 \mathrm{BL} / 6$ background. Mice were maintained on a standard rodent diet (formula LM-458, 7912; Harlan Teklad) containing $284 \mathrm{mg}$ iron $/ \mathrm{kg}$. In certain experiments, mice were fed a low-iron (3-6 mg/kg food) diet (Formula TD 80396; Harlan Teklad) for 3 weeks.

Hematological analysis. Blood was obtained by retro-orbital bleeding and collected in EDTA-K2 tubes (CapiJect T-MLH; TERUMO). Blood cell counts and erythrocyte parameters were determined using a MAXM Coulter (Beckman Coulter) automatic analyzer.

Immunohistochemistry. Tissues were fixed in $10 \%$ formalin sectioned in paraffin, then subjected to microwave antigen retrieval and immunohistochemistry (Vector Blue Alkaline Phosphatase Kit; Vector Laboratories) with primary antibodies against ferroportin (Alpha Diagnostic International) at 1:200. Iron detection was performed with Perls Prussian blue and nuclear red counter stain. 
Iron quantification. Tissue iron was quantified colorimetrically by a modification of the method of Torrance et al. (33). Fifty- to 100-mg liver extracts were digested overnight in $100 \mu \mathrm{l} 3 \mathrm{M} \mathrm{HCl} / 10 \%$ trichloroacetic acid at $65^{\circ} \mathrm{C}$, followed by the addition of $200 \mu \mathrm{l}$ chromogen $(0.01 \%$ bathophenanthroline sulfonate, $0.1 \%$ thioglycolic acid, and $4 \mathrm{M}$ sodium acetate). For a standard curve, serial dilutions of a ferric iron standard (Sigma-Aldrich) were used. Color was allowed to develop for 15 minutes and measured at $535 \mathrm{~nm}$.

$R T$ and real-time quantitative PCR. First-strand synthesis was obtained from $1 \mu \mathrm{g}$ of total RNA isolated with TRI reagent (Molecular Research Center Inc.) by the SuperScript system (Invitrogen) employing random primers. For real-time PCR analyses, cDNAs were diluted to a final concentration of $10 \mathrm{ng} / \mu \mathrm{l}$ and amplified in a SYBR Green TaqMan Universal Master Mix (Applied Biosystems). Fifty nanograms cDNA was used as template to determine the relative amount of mRNA by real-time PCR in triplicate (ABI Prism 7700 sequence detection system; Applied Biosystems), using specific primers with the following sequences: hepcidin forward, 5'-TGTCTCCTGCTTCTCCTCCT-3'; hepcidin reverse, 5'-CTCTGTAGTCTGTCTCATCTGTTG-3'; ferroportin forward, 5'-CTACCATTAGAAGGATTGACCAGCTA-3'; ferroportin reverse, 5'-ACTGGAGAACCAAATGTCATAATCTG-3'; EPO forward, 5'-AATGGAGGTGGAAGAACAGGCCAT-3'; EPO reverse, 5'-CGAAGCAGTGAAGTGAGGCTACGTA-3'; $\beta$-actin forward, $5^{\prime}$ AGGCCCAGAGCAAGAGAGG-3'; $\beta$-actin reverse, $5^{\prime}$-TACATGGCTGGGGTGTTGAA-3'.

ChIP assay. For the in vivo ChIP assay, approximately $130 \mathrm{mg}$ of livers from WT and $\mathrm{VHL}^{-/-}$mice were extracted and submitted to the protocol developed by Farnham's laboratory and described at http://genomecenter. ucdavis.edu/farnham/farnham/protocols/tissues.html.

Primers flanking the putative HREs within the murine Black6 Hepc promoter were: -2366-mHEPC-F, 5'-CCATCACTTATAGTGGGCTC-3', and -1915-mHEPC-R, 5'-GCCATTGTCACACAGCTACA-3'.

Western blot studies. Livers were harvested, ground in a mortar, and washed with PBS and proteins extracted with RIPA buffer. Twenty micrograms of total (for ferritin and ferroportin) or nuclear extracts (for hepcidin and HIF) were loaded on a $10 \%$ (ferritin and ferroportin), $12 \%$ (hepcidin), or $7 \%$ (HIF) gels, and Western blot analysis was performed using standard methodologies. Primary antibodies were rabbit anti-mouse Hepcidin (Alpha Diagnostic International), rabbit anti-HIF- $1 \alpha$ (Novus Biologicals), rabbit anti-ferroportin (Alpha Diagnostic International), and rabbit anti-ferritin heavy chain (Abcam) at a concentration of 1:1,000.

Transient transfection and luciferase assay. The hepcidin promoter luciferase reporter plasmid (pGL3-HRE) was constructed by cloning a PCR fragment of the human hepcidin upstream region (-762 bp to $-20 \mathrm{bp}$ ) into a pGL3 vector (Promega) by conventional cloning procedures to yield pGL3-Hepc/HRE. A mutation of the putative HRE, in which the CG in the CACGTG motif was replaced by an A, was generated by site-directed mutagenesis using the QuikChange Site-Directed Mutagenesis kit (Stratagene) as indicated by the manufacturer. The point mutation in the corresponding plasmid pGL3-Hepc/mutHRE was verified by sequencing. HEK293 cells were transfected by Lipofectamine 2000 (Invitrogen) with $2 \mu \mathrm{g}$ of pGL3, pGL3-Hepc/HRE, or pGL3-Hepc/mutHRE, according to the manufacturer's protocol. The following day, cells were incubated with DFO at $150 \mu \mathrm{M}$ for 18 hours. Cells were then washed with PBS and lysed with the reporter gene assay lysis buffer (Roche), and luciferase assay was performed using the Bright-Glo Luciferase Assay kit (Promega).

Statistics. All values in the figures are expressed as mean \pm SD. Student's $t$ test (unpaired, 2 tailed) was used for comparison between experimental groups. A $P$ value of less than 0.05 was considered significant.

\section{Acknowledgments}

This work was supported by a grant from the NIH (AI060840 to R.S. Johnson and V. Nizet) and the Swiss National Foundation (PBZHB108365 to A.S. Zinkernagel). We gratefully acknowledge Nissi Varki for her histopathology expertise, Wen Liu for his help with cloning procedures, and Charlene Barroga for assistance with vector construction and use of luminometer.

Received for publication December 29, 2006, and accepted in revised form April 10, 2007.

Address correspondence to: Victor Nizet, Department of Pediatrics, UCSD School of Medicine, 9500 Gilman Drive, MC-0687, La Jolla, California 92093-0687, USA. Phone: (858) 534-7408; Fax: (858) 534-5611; E-mail:vnizet@ucsd.edu. Or to: Randall S.Johnson, Molecular Biology Section, UCSD School of Medicine, 9500 Gilman Drive, MC-0377, La Jolla, California 92093-0377, USA. Phone: (858) 822-0509; Fax: (858) 822-5833; E-mail: rsjohnson@ucsd.edu.
1. Nemeth, E., et al. 2004. Hepcidin regulates cellular iron efflux by binding to ferroportin and inducing its internalization. Science. 306:2090-2093.

2. Nicolas, G., et al. 2002. Severe iron deficiency anemia in transgenic mice expressing liver hepcidin. Proc. Natl. Acad. Sci. U. S. A. 99:4596-4601.

3. Nicolas, G., et al. 2001. Lack of hepcidin gene expression and severe tissue iron overload in upstream stimulatory factor 2 (USF2) knockout mice. Proc. Natl. Acad. Sci. U. S. A. 98:8780-8785.

4. Lee, P., Peng, H., Gelbart, T., Wang, L., and Beutler, E. 2005. Regulation of hepcidin transcription by interleukin-1 and interleukin-6. Proc. Natl. Acad. Sci. U. S. A. 102:1906-1910.

5. Nemeth, E., et al. 2004. IL-6 mediates hypoferremia of inflammation by inducing the synthesis of the iron regulatory hormone hepcidin. J. Clin. Invest. 113:1271-1276. doi:10.1172/JCI200420945.

6. Weinstein, D.A., et al. 2002. Inappropriate expression of hepcidin is associated with iron refractory anemia: implications for the anemia of chronic disease. Blood. 100:3776-3781.

7. Nicolas, G., et al. 2002. The gene encoding the iron regulatory peptide hepcidin is regulated by anemia, hypoxia, and inflammation. J. Clin. Invest. 110:1037-1044. doi:10.1172/JCI200215686.
8. Feder, J.N., et al. 1996. A novel MHC class I-like gene is mutated in patients with hereditary haemochromatosis. Nat. Genet. 13:399-408.

9. Muckenthaler, M., et al. 2003. Regulatory defects in liver and intestine implicate abnormal hepcidin and Cybrd1 expression in mouse hemochromatosis. Nat. Genet. 34:102-107.

10. Papanikolaou, G., et al. 2005. Hepcidin in iron overload disorders. Blood. 105:4103-4105.

11. Roetto, A., et al. 2003. Mutant antimicrobial peptide hepcidin is associated with severe juvenile hemochromatosis. Nat. Genet. 33:21-22.

12. Semenza, G.L. 2001. HIF-1 and mechanisms of hypoxia sensing. Curr. Opin. Cell Biol. 13:167-171.

13. Flamme, I., et al. 1997. HRF, a putative basic helixloop-helix-PAS-domain transcription factor is closely related to hypoxia-inducible factor- 1 alpha and developmentally expressed in blood vessels. Mech. Dev. 63:51-60.

14. Ema, M., et al. 1997. A novel bHLH-PAS factor with close sequence similarity to hypoxia-inducible factor 1alpha regulates the VEGF expression and is potentially involved in lung and vascular development. Proc. Natl. Acad. Sci. U. S. A. 94:4273-4278.

15. Tian, H., McKnight, S.L., and Russell, D.W. 1997. Endothelial PAS domain protein 1(EPAS1), a transcrip- tion factor selectively expressed in endothelial cells. Genes Dev. 11:72-82.

16. Gu, Y.Z., Moran, S.M., Hogenesch, J.B., Wartman, L., and Bradfield, C.A. 1998. Molecular characterization and chromosomal localization of a third alpha-class hypoxia inducible factor subunit, HIF3alpha. Gene Expr. 7:205-213.

17. Frazer, D.M., et al. 2002. Hepcidin expression inversely correlates with the expression of duodenal iron transporters and iron absorption in rats. Gastroenterology. 123:835-844.

18. Kemna, E., Pickkers, P., Nemeth, E., van der Hoeven, H., and Swinkels, D. 2005. Time-course analysis of hepcidin, serum iron, and plasma cytokine levels in humans injected with LPS. Blood. 106:1864-1866.

19. Bhatia, S.N., Balis, U.J., Yarmush, M.L., and Toner, M. 1999. Effect of cell-cell interactions in preservation of cellular phenotype: cocultivation of hepatocytes and nonparenchymal cells. FASEB J. 13:1883-1900.

20. Rankin, E.B., et al. 2005. Inactivation of the arylhydrocarbon receptor nuclear translocator (Arnt) suppresses von Hippel-Lindau disease-associated vascular tumors in mice. Mol. Cell. Biol. 25:3163-3172.

21. Haase, V.H., Glickman, J.N., Socolovsky, M., and Jaenisch, R. 2001. Vascular tumors in livers with 
targeted inactivation of the von Hippel-Lindau tumor suppressor. Proc. Natl. Acad. Sci. U. S. A. 98:1583-1588.

22. Ang, S.O., et al. 2002. Disruption of oxygen homeostasis underlies congenital Chuvash polycythemia. Nat. Genet. 32:614-621.

23. Gordeuk, V.R., et al. 2004. Congenital disorder of oxygen sensing: association of the homozygous Chuvash polycythemia VHL mutation with thrombosis and vascular abnormalities but not tumors. Blood. 103:3924-3932.

24. Wenger, R.H., and Gassmann, M. 1997. Oxygen(es) and the hypoxia-inducible factor-1. Biol. Chem. 378:609-616.

25. Abboud, S., and Haile, D.J. 2000. A novel mammalian iron-regulated protein involved in intracellular iron metabolism. J. Biol. Chem. 275:19906-19912. 26. Viatte, L., et al. 2005. Deregulation of proteins involved in iron metabolism in hepcidin-deficient mice. Blood. 105:4861-4864.

27. Huang, F.W., Pinkus, J.L., Pinkus, G.S., Fleming, M.D., and Andrews, N.C. 2005. A mouse model of juvenile hemochromatosis. J. Clin. Invest. 115:2187-2191. doi:10.1172/JCI25049.

28. Mazure, N.M., et al. 2002. Repression of alpha-fetoprotein gene expression under hypoxic conditions in human hepatoma cells: characterization of a negative hypoxia response element that mediates opposite effects of hypoxia inducible factor- 1 and c-Myc. Cancer Res. 62:1158-1165.

29. Chen, K.F., Lai, Y.Y., Sun, H.S., and Tsai, S.J. 2005. Transcriptional repression of human cad gene by hypoxia inducible factor-1alpha. Nucleic Acids Res. 33:5190-5198.

30. Yoon, D., et al. 2006. Hypoxia-inducible factor-1 deficiency results in dysregulated erythropoiesis signaling and iron homeostasis in mouse development. J. Biol. Chem. 281:25703-25711.

31. Brugnara, C., et al. 1994. Effects of subcutaneous recombinant human erythropoietin in normal subjects: development of decreased reticulocyte hemoglobin content and iron-deficient erythropoiesis. J. Lab. Clin. Med. 123:660-667.

32. Finch, C. 1994. Regulators of iron balance in humans. Blood. 84:1697-1702.

33. Torrance, J.D., Charlton, R.W., Schmaman, A., Lynch, S.R., and Bothwell, T.H. 1968. Storage iron in "muscle". J. Clin. Pathol. 21:495-500. 